replacement costs; and b) measurement of perceived financial burden without an attempt to quantify that burden. Financial burden was associated with longer caregiving experiences and caregiving for people with dementia, but there was conflicting evidence about effects among financially vulnerable populations such as caregivers of color. Very few studies sought to quantify the costs of caregiving, and findings offered a wide range of actual costs. Gaps in the literature will be identified with discussion of implications and directions for future research.

\section{MEMORIAL SOCIAL WORK FOR THE DEAD AND THEIR LIVING FAMILIES: MAPPING THE FIELD AND RESEARCH AGENDA}

H. Park, Western Sydney University, Sydney, New South Wales, Australia

Death is likely to be regarded as a taboo topic in social work with older people. When it occurs, client engagement does come to an end. There is also an insufficiency of intercultural understanding about posthumous reputations and ancestor remembrance in the social work context. Challenging such a prevailing 'passive' approach to death and the dead, this paper discusses how the practice of ancestor respect was perceived and experienced among Korean people living in different regional settings. Data were collected from interviews with 61 participants, mainly older people and social workers, from Korea, New Zealand and Australia, and then analysed using a thematic analysis approach in the bilingual context in which two languages, Korean and English, were used interchangeably.

The findings from this qualitative study show that ancestors and other late relatives are important parts of people's lives and relationships in Korean culture. Most participants perceived ancestor respect as a core aspect of filial piety requiring some action, not necessarily worship but more likely an expression of veneration and remembrance. This traditional filial practice has allowed family members to gather together to share memories, and maintain connections with relatives and ancestors. The thematic analysis of findings indicates that the memorial aspect of filial piety has significant implications for social work practice, training and research. A potential area of social work, namely 'memorial social work', is proposed to cover the issues concerning death and the hereafter, such as images of death and ancestors, loss and grief, after-life planning and legacy-helping practice.

\section{FILIAL PIETY (XIAO): PERSPECTIVES ON INSTITUTIONAL ELDER CARE AMONG COLLEGE STUDENTS IN CHINA}

\section{J. Tan, Bridgewater State University, Bridgewater,} Massachusetts

China has the largest net aging population in the world along with a rapid aging pace. Historically, the family has been the source of support and care for their elders, and filial piety has been a fundamental tenet in Chinese culture. However, increased geographic mobility and reduced family size have made more adult children unavailable for elder care. Very little empirical research has examined the perspectives among college students in China towards institutional elder care.
A mixed method approach was used with both quantitative and qualitative data collection. A convenience sample $(\mathrm{N}=380)$ was collected through anonymous, self-administered survey questionnaires. In addition three one-hour long English focus groups were conducted. Survey data were inputted and analyzed using SPSS. Focus groups were transcribed and analyzed using content analysis methods.

Results from questionnaires found that $35.4 \%$ of the participants would choose institutional care for their parents, and $15 \%$ more $(50.4 \%)$ of the participants would choose institutional care for themselves. Results from focus groups suggests filial piety, although considered very important and defined in traditional terms of obey, respect and duty, may have new interpretations of how it is practiced for the younger generation. In addition, how to care for elder parents reflects these new interpretations by including possibilities of hired and institutional care.

Findings from this study provide important and meaningful information for gerontological educators, service providers, and policy makers. With the one-child policy in place for a generation, China is facing an ever-growing need for trained professional service providers to address the needs of older adults, and institutional elder care is likely to become a more acceptable option for parent care.

\section{POWER AND AMBIVALENCE IN INTERGENERATIONAL COMMUNICATION: DECIDING TO INSTITUTIONALIZE IN SHANGHAI}

L. Chen ${ }^{1}$, L. Levy-Storms ${ }^{2}$, 1. Fudan University, Shanghai, China, 2. University of California Los Angeles, Los Angeles, California

Filial piety or China's tradition of taking care of one's aging parents continues to evolve as evidenced by a growth in nursing home residents in Shanghai. The reason for this increase in institutional care remains unclear and calls for an exploration on how these elders and their children decided to institutionalize. More specifically, understanding the communication dynamics between generations when they decide to institutionalize would provide insights into whether and how the decision is mutual. Using a phenomenological approach, this study draws on power relations to examine intergenerational communication dynamics during the decision-making process around institutionalization. Twelve matched dyads of elderly residents and their children participated in face-toface, in-depth interviews in a government-sponsored nursing home in Shanghai $(N=24)$. Both generations reported how they proposed to institutionalize, initiated intergenerational communication, and finalized the decision, as well as how they reacted to the other generation's stance during the decision-making process. The findings reveal that more children made the decision for their elderly parents (decision-making power) than did their frail parents for themselves. Adult children's stronger power evoked their elderly parents' ambivalent feelings of filial piety. Older adults were simultaneously disappointed about and obliged to their children's decision to institutionalize. Furthermore, in the power trajectory, one difference may exist that the children's end matches up with tangible caregiving resources, whereas the elders' stay at the emotional end. The ongoing aging of Chinese baby boomers requires future research on longitudinal caregiving trajectories between generations. This study also illuminates the 
needs for comparisons with caregiving expectations between generations to inform the development of long-term care infrastructure in urban China.

\section{WORK MADE (IN)VISIBLE: MAPPING INTERSECTIONS OF INFORMATION WORK AND CARE WORK}

N. Dalmer, The University of Western Ontario, London, Ontario, Canada

With government endorsement of aging in place, a growing reliance on family caregivers who provide unpaid care for aging family members prompts further investigation. Seeking, interpreting, creating, and sharing information (examples of information work) are one such facet of unpaid care. Information work saturates every facet of caregiving, providing tools for coping, problem solving, and dealing with uncertainty through knowledge, affirmation, and support.

Data from three separate institutional ethnographic studies trace the degree to which family caregivers' information work are made invisible or obscured in policy, scholarship, and by caregivers themselves. Findings from interviews with family caregivers of community-dwelling older adults living with dementia, analyses of aging in place policies, and a scoping review of caregiving literature collectively map out the social organization of informal caregivers' information work. In each study, an institutional ethnographic approach is implemented, examining usages of language and power, organizational interests, and indications of unstated assumptions to uncover how families' own discourses, policy documents, and academic thinking regulate understandings of caregivers' information work.

Findings from this study signify an innovative shift in the ways the complexities of searching for and using information on behalf of an aging family member are understood. Findings speak to the trend towards informatization of care, that is, how government and health care systems are using the provision of information to gradually marginalize care. Results of this study bring visibility to the concept of information work as a means to more responsively support the evolving information needs of family caregivers.

\section{SESSION 4465 (POSTER)}

\section{DELIRIUM}

\section{EFFECTIVENESS OF NON-PHARMACOLOGICAL INTERVENTION IN PREVENTING ACUTE DELIRIUM IN OLDER INPATIENTS}

\section{S. Wong, D. Yeung, Pamela Youde Nethersole Eastern}

Hospital, Hong Kong, Hong Kong

Delirium is a complex neuropsychiatric syndrome characterized by acute onset of disturbance of consciousness, fluctuation of cognition and attention, and perceptual disturbance, and it is common in older hospitalized patients. It has adverse impacts on patients and healthcare outcomes: longer hospital stays, cognitive and functional decline and increased institutionalization rate. Up to one third of delirium in older patients could be prevented.

Patients in geriatric wards in a regional HKSAR hospital who were at risk of developing acute delirium were recruited.
They were randomized either into the intervention or the control group. The intervention was led by an Advance Practice Nurse (APN), and it comprised of education to family caregivers about delirium, reality orientation, correction of sensory deprivation, and the control group would receive usual care. The primary outcome was occurrence of delirium. The secondary outcomes were length of stay, fall, use of physical restraint and 28-day unplanned readmission rate after hospital discharge.

Fifty four patients were studied, 27 in the intervention group and 27 in the control group. The average age was 80.9. No patient in the intervention group developed acute delirium, while 3 patients $(11 \%)$ developed delirium in the control group, but the difference was not statistically significant $(p=0.236)$. There was no difference in the length of stay in both groups, and there was no incident of fall or use of physical restraint in both groups. The unplanned readmission rate was $22.2 \%$ in the intervention group and $37.0 \%$ in the control group $(p=0.186)$.

\section{EVALUATION OF A BRIEF PROGRAM FOR DELIRIUM ASSESSMENT AND INTERVENTION}

D. D’Avolio ${ }^{1}$, K. Barba ${ }^{2}$, P. Fitzgerald ${ }^{2}$, L. Carraci ${ }^{2}$, A.J. Guarino ${ }^{3}$, 1. Florida Atlantic University, Boca Raton, Florida, 2. Massachusetts General Hospital, Boston, Massachusetts, 3. MGH Institute of Health Professions, Boston, Massachusetts

Delirium is a serious health issue which is under recognized and misdiagnosed. It can lead to increased healthcare costs, morbidity, mortality, use of restraints and falls. The purpose of this study was to determine the feasibility of a brief educational intervention on nurses' delirium knowledge, assessment and intervention skills. We used a quasiexperimental, one-group pretest-posttest quasi-experimental design $\left(\mathrm{O}_{1} \mathrm{X} \mathrm{O}_{2}\right)$ design.

One hundred percent of the nurses reported a lack of delirium education in their core curriculum and lack of delirium knowledge. The educational intervention consisted of a delirium packet distributed prior to attending a one hour program. After the intervention, nurses participated in bedside teaching rounds and demonstrated delirium assessment and interventions on three patients.

Results-52 nurses screened 643 patients for delirium. During the initial screenings, $12 \%$ of patients were found positive for delirium. Patients diagnosed with delirium compared to non-diagnosed patients were statistically significantly older $\left(M_{\text {delirium }}=79.41, S D_{\text {delirium }}=11.02\right.$ and $M$ non- $^{\text {delirium }}=64.16, S D$ non- $\left.{ }_{\text {delirium }}=16.28\right)$. Logs were kept of evidence based non-pharmacological interventions. Interventions were grouped into categories; review of medications, diagnostic tests, orientation, sensory, nutrition, toileting, sleep, pain, mobility, social needs, safety, and consultation. Over 3,000 non-pharmacological interventions were implemented on patients found to be delirium positive and at risk.

The lack of delirium education in core nursing curriculum is an area that has not yet been addressed. Additionally, these results have implications for healthcare organizations to provide delirium education and practice guidelines to improve patient care. 